\title{
Mitos del amor romántico y violencia de género en jóvenes estudiantes de la Universidad del Azuay
}

\section{Myths of romantic love and gender violence in young students of the University of Azuay}

\author{
Mónica Palacios $^{1 *}$ y Paula Valverde ${ }^{1}$ \\ ${ }^{1}$ Universidad del Azuay, Ecuador \\ *monipalas@gmail.com
}

DOI: https://doi.org/10.26871/killkanasocial.v4i3.665

\begin{abstract}
Resumen
La presente investigación tuvo como objetivo identificar la relación entre los mitos del amor romántico y la violencia de género durante el noviazgo en estudiantes de la Universidad del Azuay. Se realizó un estudio experimental transversal con alcance descriptivo correlacional. Participaron 166 estudiantes, que se encontraban en una relación amorosa y que cursaban segundo año de las carreras pertenecientes a la Facultad de Ciencia y Tecnología, y a la de Filosofía, Letras y Ciencias de la Educación. La evaluación se realizó mediante la "Escala de mitos sobre el amor" y el "Cuestionario de Violencia entre Novios" (CUVINO). Los datos se analizaron mediante el programa estadístico SPSS 25, y la edición de tablas y gráficos en Excel 2019. Los resultados se presentaron mediante medidas de tendencia central y dispersión; el comportamiento de datos según la prueba Kolmogorov Smirnov resultó no normal, por lo que se emplearon pruebas no paramétricas; la prueba de comparación de medias para dos grupos U- Mann Whitney y la prueba H-Kruskal Wallis para más de dos grupos, además se empleó el coeficiente de correlación Rho de Spearman. El análisis de resultados no evidenció una correlación significativa estadísticamente entre los mitos del amor romántico y la violencia de género en el noviazgo, sin embargo, la interiorización de los mitos estuvo presente en la mayoría de la población estudiada en diversos grados; y un bajo porcentaje de la muestra había sido víctima de violencia en el noviazgo, por lo que se corrobora la hipótesis planteada.
\end{abstract}

Palabras clave: amor romántico, género, mitos, noviazgo, violencia.

\begin{abstract}
The present research aimed to identify the relationship between myths of romantic love and gender violence during dating in young students of the University of Azuay. A cross- sectional and experimental study with a descriptive and correlational scope was carried out. The participants were 166 students, who were in a romantic relationship during the investigation and who were in their second year of studies at the Faculties of Science and Technology, and Philosophy, Letters and Education Sciences. The evaluation was carried out through the "Scale of myths about love" and the "Questionnaire on Couple Violence" (CUVINO). The data were analyzed using the SPSS 25 statistical program, and the tables and graphs edition in Excel 2019. The results were presented using measures of central tendency and dispersion; the behavior of the data according to the Kolmogorov Smirnov test was non-normal $(p<.05)$, so non-parametric tests were used, the test of comparison of means for two groups U-Mann Whitney and the H-Kruskal Wallis test for more of two groups; In addition, the Spearman Rho correlation coefficient was used. After using the aforementioned methods, the results showed that, although there was no statistically significant correlation between the myths of romantic love and gender violence in dating, the internalization of myths was present in the majority of the participants at different levels; and a low percentage of the sample had been a victim of dating violence, which corroborated the hypothesis raised.
\end{abstract}

Keywords: gender, myths, romantic love, dating, violence.

\section{Introducción}

En los dos últimos siglos el amor ha adquirido un papel central en la configuración del ser humano. (Esteban y Távora, 2008). El amor es una construcción humana compleja compuesta por una dimensión social y cultural, que influyen, modelan y determinan nuestras relaciones eróticas y afectivas, metas, anhelos, etc. Esta emoción surge en cada ser humano desde niños, y se construye en base a la moral, normas, tabúes, costumbres, creencias, y necesidades de cada sistema social, por eso cambia en el tiempo y espacio $(?, ?)$. 
Robert J. Sternberg (1986, 1988, 1989) describió al amor desde su estructura y dinámica, y señaló a la intimidad, pasión y compromiso como sus componentes principales; siendo la combinación de estos tres lo que provoca diferentes clases de amor (Ferrer Pérez y Bosch Fiol, 2012). Desde esta perspectiva, el amor romántico está compuesto por intimidad y pasión, y carece de compromiso, volviéndolo difícil para mantener una relación estable a través del tiempo. Este amor romántico, se configura en un contexto socio cultural determinado como consecuencia de la llamada socialización diferencial (Giddens, 2001).

La sociabilización diferencial explica que niños y niñas son diferentes por naturaleza, por ende, se espera que desempeñen diferentes roles en su vida adulta. Así pues, hombres y mujeres, socializados de manera diferencial en el contexto de una sociedad patriarcal, entienden por amor y amar cosas diferentes (Ferrer Pérez y Bosch Fiol, 2012).

Según Marroquí y Cervera (2014) el amor romántico nace en Europa como una construcción social del amor y de la familia, para definir los roles que cada uno debe tener. Algunas características de este amor son vivir experiencias muy intensas de felicidad o de sufrimiento, depender de la otra persona, adaptarse a ella y postergar lo propio, perdonar y justificar todo en nombre del amor, entre otras.

Tanto la sociabilización diferencial como la creencia en el amor romántico, han generado una serie de ideas fijas sobre lo que las personas deben dar y recibir cuando se interrelacionan. Estas ideas en muchas ocasiones están vinculadas con lo que se conoce como mito, los cuales otorgan modelos de conducta y actitudes a la sociedad (?, ?).

Se puede decir que, un mito es una creencia formulada como una verdad y expresada de forma absoluta y poco flexible que suele poseer carga emotiva y busca contribuir a crear y mantener la ideología del grupo, suelen ser resistentes al cambio y al razonamiento lógico, aunque cambian entre culturas adaptándose a la realidad socioeconómica y política de cada una (Bosch y cols., 2007).

De la unión entre estas ideas fijas llamadas mitos, y el concepto de amor romántico, surge en Occidente los conocidos "mitos del amor romántico (Sanpedro, 2004). Yela (2019) define a los mitos del amor romántico como el conjunto de creencias socialmente compartidas sobre la "supuesta verdadera naturaleza del amor", y los describe como ficticios, absurdos, engañosos, irracionales e imposibles de cumplir. Puede decirse que han sido desarrollados con el objetivo de primar un determinado modelo de relación monógama y heterosexual.

Estos mitos suelen generar altas expectativas que, a su vez provocan consecuencias personales y sociales como frustración, sufrimiento, insatisfacción, desaprobación (Ferrer, Bosch, y Navarro, 2010), promueven también aquello que nos separa y aleja de la anhelada igualdad de género, interrumpe la potencialización y desarrollo de cada ser humano; y en muchos casos suscitan actitudes violentas que lastimosamente llegan a normalizarse.
Investigadores han concentrado su foco de interés en el análisis de la presencia de conductas violentas en relaciones de noviazgo entre jóvenes, ya que se entiende que son las predecesoras de indicadores de violencia en relaciones futuras. Como resultado, existen muchos datos que avalan que el comienzo de diversas manifestaciones de violencia suele darse en las parejas jóvenes, donde tanto la agresión psicológica como la física forman parte de las relaciones interpersonales y en muchos casos, estos comportamientos son considerados como una práctica «normal» dentro de la pareja (Muñoz-Rivas, Graña Gómez, O’Leary, y González Lozano, 2007).

De acuerdo con los estudios disponibles, hasta un $50 \%$ de jóvenes han ejercido alguna forma de agresión física contra su pareja y un porcentaje aún mayor informa haber empleado agresión psicológica (Muñoz-Rivas, GámezGuadix, Graña, y Fernández, 2009). Marina Muñoz señala que las personas empezamos a tener nuestras primeras relaciones a los 12 años aproximadamente, y es ahí cuando diversas formas de agresión comienzan a estar presentes en el $90 \%$ de dichas relaciones, provocando en los seres humanos el desarrollo de elementos explicativos determinantes para todos estos acontecimientos, dándose así el proceso de habituación psicológica (Muñoz Rivas, 2019).

La violencia intrafamiliar no tiene su origen al momento en que se contrae matrimonio; sino tiene un antecedente inmediato como la elección de pareja y noviazgo (Olvera, López, y Velazquez, 2012). Dicha situación es a futuro un factor determinante en la desintegración del núcleo familiar o de la perpetuación de este modelo de conducta. Se reconoce entonces que es necesario intervenir en la modificación de esas pautas de comportamiento violento.

En el Ecuador se ha logrado visibilizar la violencia como un problema de salud pública debido a la lucha de las mujeres, lo que ha generado un interés a nivel político. Esta visibilidad se acentuó más cuando la Encuesta Nacional de Relaciones Familiares y Violencia de Género contra las mujeres realizada en el 2011, reveló que, en el Ecuador, 6 de cada 10 mujeres habían sido víctimas de violencia, es decir, más de 3.2 millones de mujeres han sufrido algún tipo de violencia por el hecho de ser mujeres. También demostró que, 1 de cada 4 mujeres ha vivido violencia sexual; sin embargo, la violencia psicológica es la forma más recurrente de violencia de género. En el $76 \%$ de los casos de violencia de género contra las mujeres, el agresor ha sido su pareja o ex pareja (Asamblea Nacional República del Ecuador, 2018). Es así que en el 2014 se incluye en el Código Orgánico Integral Penal al femicidio como delito y en el 2018 se publica la Ley orgánica Integral para Prevenir y erradicar la violencia contra las mujeres en donde establece 3 componentes como la atención, protección y reparación de las mujeres para la erradicación de la misma (Asamblea Nacional República del Ecuador, 2018).

La Organización Mundial de la Salud (OMS, s.f.) divide la violencia en tres categorías generales: violencia auto infligida, violencia interpersonal y violencia colectiva. 
El artículo 10 de la Ley Orgánica Integral para prevenir y erradicar la violencia contra las mujeres describe diferentes tipos de violencia como: física, psicológica, sexual, económica y patrimonial, simbólica, política y gineco-obstétrica.

\section{Marco Teórico}

Los mitos del amor romántico juegan un papel importante dentro de las interacciones de los seres humanos, especialmente en las relaciones de pareja. La interiorización de estos, muchas veces genera ciertas manifestaciones de violencia que lamentablemente llegan a normalizarse por la falta de concientización sobre el tema.

La violencia de género que puede evidenciarse en la actualidad, es considerada un problema social y de salud pública que atenta en contra de los derechos humanos y dentro de la cual nos vemos inmersos en muchas ocasiones sin percibir la magnitud de la misma.

No existen estudios relacionados a esta temática en nuestro medio, es por esto que se considera necesario tomar en cuenta las investigaciones realizadas a nivel mundial y contextualizarlas en nuestra realidad. Corral (2009) señala que las tasas de violencia son superiores en cuanto a la perpetración por parte de los varones y existe una mayor victimización en las mujeres. La Organización Mundial de la Salud (2013) indica que alrededor de tres de cada diez adolescentes denuncian ser víctimas de violencia en el noviazgo. Así también, José Luis Rojas y Eugenio Carpintero Raimundez (2015), hallaron diferencias significativas en los niveles de sexismo entre hombres y mujeres, siendo los hombres quienes han cometido más agresiones sexuales, mientras que las mujeres más agresiones verbales y emocionales, y no se encontraron diferencias en cuanto a agresiones físicas. Según López-Cepero (2015), en España el 3,6\% de los hombres y el 7,9\% de las mujeres se han sentido víctimas de maltrato durante alguna relación de noviazgo juvenil.

Estos son algunos de los datos estadísticos que motivaron la realización de este estudio, ya que teniendo una visión global de la problemática se considera que son los jóvenes lo más beneficiados con esta investigación, ya que al dar a conocer el tema a profundidad y las causas que lo generan, se espera generar conciencia de la gravedad y realidad del problema, pudiendo llegar a crear medidas que prevengan este tipo de conductas tan normalizadas hoy en día.

\section{Metodología}

\subsection{Tipo de estudio}

El presente estudio es experimental, abierto, transversal, prolectivo, prospectivo con alcance descriptivo correlacional.

\subsection{Sujetos de estudio}

Participaron 166 estudiantes, que se encontraban en una relación amorosa al momento de la aplicación y que además cursaban segundo año en su carrera, pertenecientes a la Facultad de Ciencia y Tecnología, así como a la de Filosofía, Letras y Ciencias de la Educación de la Universidad del Azuay. Se tomó en cuenta el número de relaciones anteriores y el tiempo de su relación actual.

\subsection{Mediciones, test o pruebas}

Para dar inicio a la investigación, se entregó un consentimiento informado a cada uno de los participantes del estudio, en el que autorizaron proceder con la aplicación de la encuesta sociodemográfica y los dos instrumentos escogidos. La encuesta socio-demográfica se elaboró en base a los criterios de inclusión y exclusión, así como también a las variables que se pretenden analizar. Posteriormente, se aplicaron los instrumentos psicológicos a los participantes; se inició por la "Escala de mitos sobre el amor", seguida del "Cuestionario de Violencia entre Novios (CUVINO)".

\subsection{Ficha sociodemográfica}

Mediante este instrumento se recogieron algunos datos de los participantes tales como género, edad, carrera en la que están matriculados, religión, estado civil, número de relaciones amorosas a lo largo de su vida, tiempo total de su relación amorosa actual.

\subsection{1 "Escala de mitos sobre el amor"}

Este es un instrumento creado por Esperanza Bosch Fiol, Victoria A. Ferrer Pérez, M. Esther García Buades, M. Carmen Ramis Palmer, M. Carmen Mas Tous, Capilla Navarro Guzmán, y Gema Torrens Espinosa quienes tomaron como punto de partida el trabajo de Ana Barrón y cols. (2007) que evalúa la vigencia de los mitos: de la "media naranja", del emparejamiento, de los celos, de la omnipotencia, del matrimonio, de la pasión eterna y de la compatibilidad entre amor y violencia. A partir de ahí, obtuvieron 10 ítems que constituyen la "Escala de mitos sobre el amor", con objeto de que estos ítems sirvieran para valorar el acuerdo de las personas entrevistadas con los mitos, se les solicitó que valoraran si estaban o no de acuerdo con las afirmaciones planteadas en una escala de respuesta de cinco puntos, donde, a mayor puntuación, mayor será el nivel de aceptación del mito en cuestión.

Estos autores validaron la escala y fijaron un tamaño muestral, para un nivel de confianza del 95,5\% y un error de $\pm 2,9 \%$, de 1.196 personas para la condición más desfavorable $(\mathrm{p}=\mathrm{q}=50 \%)$. La muestra estuvo constituida por un total de 1.351 personas, el $51.2 \%$ eran mujeres y un $48.8 \%$ varones. Por lo que se refiere a la edad, la muestra estudiada presenta una edad media de 48,63 años, en un rango de 18 a 93. Este estudio fue realizado con la población española entre el año 2004 al 2007 por investigadores de la Universidad de les Illes Balears (Bosch y cols., 2007). 


\subsubsection{Cuestionario de violencia entre novios (CUVINO)}

Este instrumento evalúa la victimización en las relaciones de noviazgo, y está dirigido a adolescentes y jóvenes. Fue validado en jóvenes hispanohablantes por Luis Rodríguez Franco, Javier López Cepero Borrero, Francisco Javier Rodríguez Díaz, Carolina Bringas Molleda, M. A. Antuña Bellerin y Cristina Estada Pineda (2010), autores pertenecientes a las universidades de Sevilla, Oviedo y Guadalajara, quienes analizaron los resultados aportados por 5170 personas escolarizadas provenientes de España, México y Argentina.

El cuestionario se compone de 42 indicadores evaluados en una escala Likert de 5 puntos (de 0 a 4 ) agrupados en ocho factores: Desapego, Humillación, Sexual, Coerción, Físico, Género, Castigo Emocional e Instrumental y tres etiquetas de tipo dicotómico (Maltrato, Miedo y Atrapamiento) que ofrecen ocho combinaciones de respuesta posibles. Específicamente, en nuestro estudio se ha hallado una fiabilidad total de la escala de 0.948 .

\section{Resultados}

Los resultados se presentan mediante medidas de tendencia central y dispersión; el comportamiento de datos según la prueba Kolmogorov Smirnov era no normal ( $\mathrm{p}$ $<.05$ ), por lo que se emplearon pruebas no paramétricas, la prueba de comparación de medias para dos grupos UMann Whitney y la prueba H-Kruskal Wallis para más de dos grupos; además para establecer las relaciones entre la edad, tiempo de relación y número de parejas se empleó el coeficiente de correlación Rho de Spearman. Las decisiones se tomaron con una significancia del $5 \%(\mathrm{p}<.05)$.

La presente investigación fue realizada con 166 estudiantes de la Universidad del Azuay, en donde participaron 99 hombres $(59.64 \%)$ y 67 mujeres $(40.36 \%)$ de entre 18 y 37 años; con un promedio de 5 relaciones amorosas a lo largo de su vida. La finalidad de este estudio fue analizar la relación existente entre mitos del amor romántico y violencia en el noviazgo; los resultados obtenidos se plantearán en un inicio por la interiorización de los mitos del amor romántico, seguido por la violencia en el noviazgo y finalmente la relación entre estos.

Esta investigación reveló que a pesar de que existe baja presencia de mitos a nivel general, el $74.7 \%$ de estudiantes estuvieron completamente de acuerdo en al menos uno y máximo 7 ítems de forma simultánea del amor romántico, a diferencia del estudio realizado por Fundación Mujeres (2011) en Andalucía que reveló que la mayoría de encuestados ha interiorizado los mitos, y respondieron al cuestionario de manera mitificada. Se debe destacar que nuestro estudio no encontró diferencia significativa en la interiorización de mitos según la etapa de enamoramiento.

El mito predominante en los estudiantes del estudio es el que refiere al emparejamiento, es decir que "se puede ser feliz sin tener una relación de pareja" con una media de 4.18 que representa un $79.52 \%$ de la población, porcentaje alto que concuerda con el estudio dirigido por Esperanza Bosch
(2007) acerca de la interiorización de los mitos del amor romántico con la población española que determinó un 68,4\%; de igual forma que el estudio realizado en México (2016) que resultó una media de 4.41. Estos resultados distan del estudio realizado por Marroquí y Cervera de la Universidad de Granada (2014) en donde solo el 13,7 \% de los participantes creen que se puede llegar a ser feliz sin tener una relación de pareja.

Seguido a este mito, se encuentra el mito de la omnipotencia, "el amor verdadero lo puede todo" con una media de 3.84 y un porcentaje de $67.47 \%$ de la población, que coincide con los estudios de Marroquí y Cervera (2014) en donde la mayoría de la población cree que se debería dar todo por amor y que el amor lo puede todo, resultado que concuerda con una investigación realizada con 362 estudiantes de Madrid por Enrique Bonilla Algovia, Esther Rivas Rivero, Bárbara García Pérez y Alberto Criado Martos (2017), quienes concluyeron que el mito de la omnipotencia mostró mayor porcentaje de acuerdo con las creencias sexistas y la tolerancia a la violencia en el interior de la pareja. De igual manera el estudio de la Fundación Mujeres (2011) concluyó que la población responde en alto grado al mito de la omnipotencia, resultados similares a los presentados por Esperanza Bosch (2007) en donde este mito representa una media de 3.94 .

Otro mito con alto porcentaje de aceptación en la presente investigación fue el mito de la media naranja: "en alguna parte hay alguien predestinado para cada persona"; con un $51.20 \%$ en la población estudiada, convirtiéndose en el cuarto mito más representativo estadísticamente.

Este resultado coincide con la investigación del equipo dirigido por Esperanza Bosch (2007) que expresó un 52.6\% de aceptación, al igual que el estudio realizado por la Fundación Mujeres (2011) el cual reveló que un $68.5 \%$ de su población se identificaba con este mito. De igual forma Marroquí y Cervera (2014) concluyeron que el $65 \%$ de la población se encontraban de acuerdo o completamente de acuerdo con este, el cual fue el más representativo según un estudio realizado en México por Luis Rodríguez y Lorena Treviño (2016).

Marroquí y Cervera (2014) comprueban en su investigación que la gran mayoría de la población rechaza la creencia de compatibilidad entre amor y violencia, resultados que concuerdan con nuestro estudio donde se evidencia que la población estudiada no supera el $7 \%$ de interiorización de los ítems referentes a este mito. A pesar del bajo porcentaje de aceptación de esta creencia, en el presente estudio los resultados reflejan a las mujeres con una media superior en relación a los hombres (Mmujeres $=1.55$; Mhombres $=1.34$ ), resultados que distan de un estudio realizado con población colombiana (2018), donde se concluye que a pesar de la baja interiorización de este mito, los hombres son quienes más puntúan en el mismo (Mmujeres =1.44; Mhombres $=1.83$ ).

La "Escala de mitos sobre el amor" utilizada en la presente investigación, agrupa los diez ítems en dos fac- 
tores: "Idealización del amor" y "Vinculación entre amor y maltrato", basándonos en esta categorización, nuestros resultados demuestran que el factor de "Idealización del amor" es más fuerte que el de "Vinculación entre amor y maltrato", similar al estudio realizado por Bonilla y Rivas con estudiantes de Colombia (2018) el cual demostró estos resultados con una media de 2.93 y de 1.55 respectivamente; resultado que dista del equipo de Esperanza Bosch (2007) quienes demostraron mayor puntuación en el factor de "Vinculación amor maltrato" $(85,6 \%)$ y en menor porcentaje al factor de "Idealización del amor" (49,2\%).

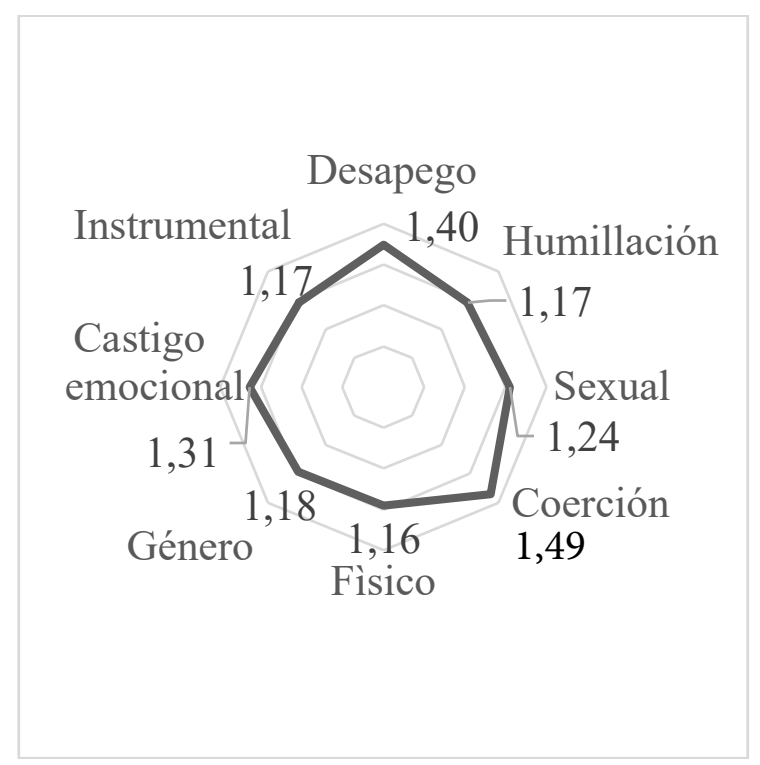

Figura 1: Factores de violencia

La presente investigación encontró que el $16.9 \%$ de estudiantes han sido víctimas al menos de una manifestación de violencia y máximo de 12 de forma simultánea, sin embargo, el porcentaje de actitudes de violencia dentro de sus relaciones amorosas es bajo. En el año 2005 Krahé y Berger concluyeron tras un estudio que un $44 \%$ de los jóvenes habían admitido ser víctimas de agresiones físicas leves alguna vez. Bajo esta línea existen otras investigaciones como la realizada en Asturias (2007), que reveló que 20.2\% de la población sufría de alguna manifestación de violencia; además Viejo en el 2012 concluyó que un $22.8 \%$ de los adolescentes estaban implicados en agresión física a sus parejas, mientras que un $29.6 \%$ lo estaban en victimización (Rodríguez y Alonso, 2015); otra investigación realizada en México por Luis Rodríguez y Lorena Treviño (2016), demostró que de los participantes el 17.14 \% había ejercido violencia física hacia su pareja, el $20 \%$ sexual, el $48.57 \%$ verbal y el $60 \%$ violencia psicológica.

Los resultados de los 8 factores de violencia evaluados revelaron que el factor predominante era el de coerción, seguido por desapego; y posteriormente el factor de castigo emocional. Estos resultados están estrechamente relacionados con la investigación realizada por Luis Rodríguez-
Franco y colaboradores (2010) con jóvenes universitarios de España, México y Argentina, el cual determinó al factor desapego como el más predominante, seguido del factor coerción. Con respecto a los valores más bajos, los resultados del presente estudio coinciden con el mencionado anteriormente, y ubica a los factores físico e instrumental como los más débiles. Este análisis de datos lleva a pensar que existe un patrón común entre las diversas poblaciones en cuanto a las manifestaciones de violencia, a pesar de la variación entre medias existe un común denominador entre poblaciones que se puede identificar gracias a la estructura del cuestionario.

De acuerdo al resultado según el sexo de los participantes, se demostró que el comportamiento se presenta de manera similar entre hombres y mujeres, sin embargo, se encontró que los hombres se mostraban con mayor victimización en los factores: sexual, castigo emocional e instrumental, mientras que el estudio realizado por Rodríguez y Treviño (2016) en México demostró que los hombres denotan mayores niveles de violencia física y psicológica que las mujeres, mientras que las mujeres mayor nivel de violencia sexual y actitudes positivas hacia la prevención de la violencia.

Con respecto a la relación entre violencia y variables sociodemográficas se puede observar que el comportamiento de manifestación de violencia en sus 8 factores es similar y bajo en hombres y mujeres; además se encontró que los hombres se mostraban con mayor victimización en los factores: sexual, castigo emocional e instrumental.

No se encontraron relaciones entre los mitos del amor romántico, la edad de los estudiantes, el número de relaciones amorosas y tiempo con la actual pareja; salvo en el mito del matrimonio, en el que se encontró una relación leve directa con la edad; es decir a mayor edad mayor intensidad de mito.

Por otra parte, el número de relaciones amorosas se relacionaba positivamente en una leve intensidad con el castigo emocional y la violencia instrumental; lo que implica que a mayor número de relaciones amorosas mayor victimización en castigo emocional y violencia instrumental. $(\mathrm{p}<.05)$.

La dimensión de mitos del amor romántico de idealización del amor se vinculó positivamente y en una leve intensidad con los factores de violencia: "Desapego" y "Humillación"; mientras que el resto de factores no tuvieron relación alguna con esta dimensión ni la de vinculación entre amor y maltrato. Además, se encontró una relación positiva leve entre la presencia total de mitos y el factor de violencia de humillación.

De acuerdo los datos obtenidos en este estudio, la religión no es un factor determinante en la interiorización de mitos del amor romántica ni en las manifestaciones de violencia, al contrario de los resultados presentados por Rodríguez y Treviño (2016) en donde los participantes que manifiestan realizar prácticas religiosas denotan menores 
niveles de violencia física, sexual y psicológica que quienes no la practican.

\section{Conclusiones y recomendaciones}

En base a los resultados obtenidos en la presente investigación, se puede concluir que la muestra seleccionada ha interiorizado los mitos del amor romántico en diversos grados, a pesar de que en términos generales no llegan a ser valores mayores a la media, se considera importante destacar que los jóvenes estudiados viven el amor desde esta perspectiva mitificada. Así también, los resultados acreditan la presencia de las diferentes manifestaciones de violencia en el noviazgo, lo que genera una relación estrecha entre los mitos asimilados y la violencia a la que cada persona está inmersa dentro del contexto de su relación amorosa. Estas manifestaciones pueden ocurrir cuando ciertos comportamientos llegan a normalizarse o justificarse dentro de la relación, distanciándose de una interacción basada en el respeto mutuo.

La hipótesis es verídica ya que existe una verdadera relación entre los mitos del amor romántico y la violencia en el noviazgo, especialmente en comportamientos relacionados con la humillación y el desapego.

Si se parte del hecho de que este amor romántico se ha creado desde una concepción patriarcal asentada en las desigualdades de género y que conlleva a que se perpetúe el sistema jerárquico, desigual, basado en la dependencia de los miembros, es importante mostrar la falsedad de esas idealizaciones que nos encierran en máscaras sociales, las que empobrecen nuestras relaciones y nos hacen sufrir ya que chocan con la realidad, generalmente menos bella y maravillosa que la fantasía amorosa.

Desde esta perspectiva del amor romántico se han generado los mitos que hemos analizado a lo largo de este estudio, los cuales están presentes no solo en nuestra sociedad si no alrededor del mundo por lo que consideramos que es nuestra responsabilidad desmitificarlos y reformularlos, con el fin de poder construir nuevas formas de interacción en donde la relación de pareja sea más positiva, recíproca, sana y activa.

Sin darnos cuenta hemos arrastrado estos mitos a lo largo del tiempo debido a que están implícitos en varias representaciones artísticas tales como el cine, la música y la literatura, lo que ha generado conceptualizaciones erróneas de lo que debería ser el amor; ha provocado también sufrimiento, decepción y frustración cuando estos no se llegan a cumplir, incluso ha permitido que se evidencien manifestaciones de violencia que pueden ir desde el castigo emocional, humillación hasta violencia física, cualquiera de ellas igual de graves ya que atentan contra la integridad y el bienestar de quien se ve afectado.

Es necesario que se desarrollen investigaciones relacionadas a los mitos del amor romántico y la violencia en el noviazgo debido a la escasa información que existe en general, pero más aún en nuestro medio. Además, se podría realizar un estudio de mayor extensión, en el cual se amplíe la muestra para que éste tenga mayor alcance y por lo tanto genere resultados más representativos, sin embargo, consideramos que nuestro estudio alcanzó los objetivos propuestos en cuanto a la población se trata.

Al ser este un tema con gran relevancia y que se ha demostrado que forma parte de una realidad para muchas parejas llega a convertirse en una problemática de carácter social que involucra no solo a la pareja sino sus creencias y costumbres, es por esto que consideramos de vital importancia generar estrategias de prevención que busquen modificar los mitos del amor romántico, reestructurar la visión ancestral heredada y facilitar herramientas que construyan interacciones sanas y motiven a un cambio positivo acerca de la manera en la que se vive el amor hoy en día.

\section{Referencias Bibliográficas}

Algovia, E. B., y Rivero, E. R. (2018). Propiedad psicométricas de la versión reducida de la escala de mitos sobre el amor en una muestra de estudiantes colombianos. Suma Psicológica.

Algovia, E. B., Rivero, E. R., Pérez, B. G., y Martos, A. C. (2017). Mitos del amor romántico y normalización de la violencia de género en adolescentes.

Asamblea Nacional República del Ecuador. (2018). Ley orgánica integral para prevenir y erradicar la violencia contra las mujeres. (Ecuador: Suplemento del registro oficial no. 175.)

Bosch, E., Ferrer, M., García, E., Ramis, M., Ms, M., Navarro, C., y Torrens, G. (2007). Del mito del amor romántico a la violencia contra las mujeres en la pareja. Madrid: Instituto de la Mujer, Ministerio de Igualdad.

de la Salud, O. M. (2013). Comprender y abordar la violencia contra las mujeres.

Esteban, M. L., y Távora, A. (2008). El amor romántico y la subordinación social de las mujeres: revisiones y propuestas. Anuario de Psicología, 39(1), 59-73. Descargado de https://digibug.ugr.es/ bitstream/handle/10481/22465/El

Ferrer, V., Bosch, E., y Navarro, C. (2010). Los mitos románticos en españa. Boletín de Psicología, 7-31.

Ferrer Pérez, V., y Bosch Fiol, E. (2012). Del amor romántico a la violencia de género. Profesorado. Revista de Currículum y Formación de Profesorado, 17(1), 105122. Descargado de https://www.redalyc . org/pdf/567/56726350008.pdf

Giddens, A. (2001). Sociología. Madrid: Alianza Editorial.

Marroquí, M., y Cervera, P. (2014). Interiorización de los falsos mitos del amor romántico en jóvenes. Reidocrea, III, 142-146.

mujeres, F. (2011). Coeducación y mitos del amor romántico.

Muñoz Rivas, M. J. (2019). Violencia en el noviazgo: desarrollo de guías de actuación preventiva para adolescentes y agentes sociales. 
Muñoz-Rivas, M. J., Gámez-Guadix, M., Graña, J. L., y Fernández, L. (2009). Violencia en el noviazgo y consumo de alcohol y drogas ilegales entre adolescentes y jóvenes españoles. Madrid, España.

Muñoz-Rivas, M. J., Graña Gómez, J. L., O’Leary, K. D., y González Lozano, P. (2007). Physical and psychological aggression in dating relationships in spanish university students. Descargado de https://repositorio.ucjc.edu/ bitstream/handle/20.500.12020/666/ Physical

Olvera, J. A., López, J. A., y Velazquez, R. A. (2012). Tipos de violencia en el noviazgo: Estudiantes universitarias de la uaem, zumpango. Mexico.

OMS. (s.f.). Informe mundial sobre la violencia y la salud. (Washington)

Rodríguez, Y., y Alonso, P. (2015). Análisis de los discursos de los y las jóvenes sobre la violencia en las relaciones de pareja. España.

Rodriguez-Franco, L. y. c. (2010). Validación del cuestionario de violencia entre novios (cuvino) en jóvenes hispanohablantes: Análisis de resultados en españa, méxico y argentina. Anuario de Psicologia Clinica y de la Salud.

Rodriguez Otero, L. M., y Treviño Martínez, L. (2016). Violencia en pareja, amor romántico y trabajo social. voces desde futuros trabajadores sociales mexicanos. Nuevo Léon, México.

Sanpedro, P. (2004). Pensamiento crítico. Descargado de http://www.pensamientocritico . org/pilsan0704.htm

Yela, C. (2019). El blog de jos $\tilde{A} \odot$, dom. Descargado de http://www.josedominante.com/ los-mitos-romanticos-segun-carlos -yela/

Recibido: 20 de septiembre de 2020

Aceptado: 27 de noviembre de 2020

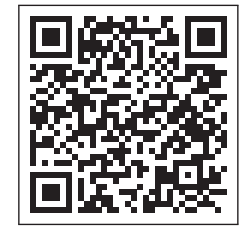


